\title{
A wavelet approach for estimating chlorophyll-a from inland waters with reflectance spectroscopy
}

\author{
Ampe Eva ${ }^{1,3}$, Erin L. Hestir ${ }^{2}$, Elga Salvadore ${ }^{1,3}$, Ludwig Triest ${ }^{4}$ and Okke Batelaan ${ }^{1,5}$ \\ Department of Hydrology and Hydraulic Engineering, VUB, Pleinlaan 2, 1050 Brussels, Belgium \\ E-mail: eva.ampe@vub.ac.be \\ 2 CSIRO, Land and Water, Canberra ACT 2601, Australia \\ 3 Flemish Institute for Technological Research (VITO), Boeretang 200, 2400 Mol, Belgium \\ 4 Department of Biology, Plant Science and Nature Management, VUB, Pleinlaan 2, 1050 Brussels, \\ Belgium \\ 5 School of the Environment, Flinders University, GPO Box 2100 , Adelaide, Australia
}

This letter presents an application of continuous wavelet analysis, providing a new semi-empirical approach to estimate Chlorophyll-a (Chl-a) in optically complex inland waters. Traditionally spectral narrow band ratios have been used to quantify key diagnostic features in the remote sensing signal to estimate concentrations of optically active water quality constituents. However, they cannot cope easily with shifts in reflectance features caused by multiple interactions of variable absorption and backscattering effects that typically occur in optically complex waters. We used continuous wavelet analysis to detect $\mathrm{Chl}-\mathrm{a}$ features at various wavelengths and frequency scales. We investigated 21 small artificial shallow ponds in the Brussels Capital Region. Here, we collected in situ pond-level reflectance spectra and related them to in situ Chl-a concentration. We used the wavelet decomposition of these spectra to build a 2D correlation scalogram with the Chl-a concentration. We isolated the most informative wavelet regions via thresholding and could relate all five regions to known inherent optical properties. We selected the optimal feature per region and compared them to three well known narrow band ratio models. The wavelet approach can provide a flexible approach to analyse high spectral resolution data because wavelets analyse the signal at different scales and synthesize information across bands. Furthermore, we hypothesize that the wavelet features are less sensitive to confounding factors such as instrument noise, coloured dissolved organic matter (CDOM) and suspended matter. 